\title{
A methyl jasmonate induced defensin like protein from Panax notoginseng confers resistance against Fusarium solani in transgenic tobacco
}

\author{
Q. WANG ${ }^{1,2}$, B.L. QIU ${ }^{1,2}$, S. LI ${ }^{1,2}$, Y.P. ZHANG ${ }^{1,2}$, X.M. CUI ${ }^{1,2}$, F. GE ${ }^{1,2}$, and D.Q. LIU ${ }^{1,2 *}$ \\ Faculty of Life Science and Technology, Kunming University of Science and Technology, Kunming, 650500, \\ Yunnan, P.R. China ${ }^{l}$ \\ Yunnan Provincial Key Laboratory of Panax notoginseng, Kunming, 650500, Yunnan, P.R. China ${ }^{2}$
}

\begin{abstract}
Plant defensins and defensin like protein (DEFL) form a large family of small cysteine-rich proteins. They are major components of plant immune systems, being involved in host defenses against biotic and abiotic stresses. In this study, a novel defensin like protein (DEFL) gene PnDEFL1 was isolated from Panax notoginseng, a traditional Chinese medicinal herb. The expression patterns of PnDEFL1 after treatment with methyl jasmonate, salicylic acid, ethephon, and $\mathrm{H}_{2} \mathrm{O}_{2}$, as well as during Fusarium solani infection, were analyzed using reverse transcription qPCR. The up-regulated expression of PnDEFL1 indicated that it responded to $F$. solani infection and all four defense-related signalling molecules. The PnDEFL1 gene was further fused with the green fluorescent protein gene in a plant expression vector and transformed into onion (Allium cepa) epidermal cells. The laser scanning confocal microscope confirmed that the PnDEFL1 protein localized to the extracellular region. In addition, the recombinant PnDEFL1 protein was expressed in Escherichia coli and purified by affinity chromatography. It had antifungal activities against $F$. solani, F. oxysporum, Botrosphaeria dothidea, and Sclerotinia sclerotiorum. The PnDEFL1 gene was transferred into tobacco (Nicotiana tabacum) to verify its function. The overexpression of PnDEFL1 in tobacco conferred a high resistance to F. solani infection. Thus, the PnDEFL1 gene is involved in the defense responses of $P$. notoginseng to $F$. solani infection.
\end{abstract}

Additional key words: Allium cepa, antifungal activity, Escherichia coli, ethephon, green fluorescent protein, $\mathrm{H}_{2} \mathrm{O}_{2}$, methyl jasmonate, Nicotiana tabacum, salicylic acid.

\section{Introduction}

In plants, biochemical and physiological responses, and even morphological lesions, occur under stress conditions, hindering normal growth and development, thereby seriously endangering agricultural production. In particular, plants are negatively affected by fungal diseases, and their large-scale occurrence usually leads to serious crop yield reductions. As a longterm response to biotic stresses, plants have gradually acquired a series of complex defense mechanisms, such as the production of plant antimicrobial peptides (AMPs) (De Souza et al. 2014). The main plant AMPs are defensins, thionins, lipid transfer proteins, cyclotides, snakins, and hevein-like proteins (Nawrot et al. 2014).
Plant defensins were first isolated from the endosperm of wheat (Triticum aestivum) and initially classified as novel glucosinolates (Colilla et al. 1990). However, they were found to have antibacterial properties and structural similarities with mammalian and insect defensins (Terras et al. 1995), and were then renamed "plant defensins". Plant defensins are cysteine-rich cationic peptides with relative molecular mass of approximately $5 \mathrm{kDa}$, containing 45 50 amino acid residues (Lacerda et al. 2014). The typical tertiary structure of a defensin is a $\operatorname{CS} \alpha \beta$ motif that has an $\alpha$-helix and three anti-parallel $\beta$-folds, and most plant defensins have eight conserved cysteine residues that form four intramolecular disulfide bonds to stabilize their tertiary structures (Fant et al. 1998, Gachomo et al. 2012). Plant defensins and defensin-like protein $(D E F L)$ genes

Submitted 10 December 2018, last revision 27 August 2019, accepted 23 September 2019.

Abbreviations: cDNA - complementary DNA; DEFL - defensin like protein; ETH - ethylene; GFP - green fluorescent protein; hpi - hours post inoculation; IPTG - isopropyl-1-thio- $\beta$-D-gac-topyranoside; JA - jasmonic acid; MeJA - methyl jasmonate; ORF - open reading frame; PDA - potato dextrose agar; RT-qPCR - reverse transcription quantitative polymerase chain reaction; SA - salicylic acid; RACE - rapid-amplification of cDNA ends; WT - wild type.

Acknowledgements: This work was financially supported by a grant received from the National Natural Sciences Foundation of China (81560610). We thank Dr. Lesley Benyon from Liwen Bianji, Edanz Group China, for editing the English text of a draft of this manuscript.

* Corresponding author e-mail: diqiuliu@126.com 
have been isolated from a variety of plants. A total of 13 defensin genes have been identified in the Arabidopsis thaliana genome (Thomma et al. 2002), and also 317 DEFLs (Silverstein et al. 2005). In total, 93, 79, and 143 DEFL genes have been characterized in rice (Oryza sativa), grapevine (Vitis vinifera), and wheat (Triticum kiharae), respectively (Silverstein et al. 2007, Giacomelli et al. 2012, Odintsova et al. 2019). In addition, the DEFLs have the hallmarks of defensin genes, including the conserved cysteine residues, CS $\alpha \beta$ motif or $\gamma$-core motif (Silverstein et al. 2005, Giacomelli et al. 2012). Moreover, the genomic organization of DEFLs is highly similar to those of mammalian defensins and plant resistance genes, and the DEFLs belong to the defensin supergene family (Silverstein et al. 2005).

The expressions of some defensin genes are regulated by pathogens, abiotic stresses, and several phytohormones. The transcription of the Capsicum annuum defensin CADEF 1 is strongly induced by Xanthomonas campestris pv. vesicatoria (Do et al. 2004). V. vinifera DEFL29 and DEFL33 are significantly up-regulated after Botrytis cinerea infection (Giacomelli et al. 2012). SlyDF1 and SlyDF2, two Solanum lycopersicum defensin genes, were strongly induced at 1 and $2 \mathrm{~d}$, respectively, after inoculation with Phytophthora infestans (Cui et al. 2018). The defensin gene $P g D 1$ of Picea glauca is up-regulated by wounding and salicylic acid (SA) treatment (Pervieux et al. 2004). The Nicotiana benthamiana NbDef2.2 is induced by wounding and responds strongly to ethylene (ETH) treatment (Bahramnejad et al. 2009). The expression of T. aestivum defensin 1 (TAD1) is induced during cold acclimation (Sasaki et al. 2016). In addition, the defensin gene $R i D$ from the wild cruciferous plant Rorippa indica is up-regulated by application of methyl jasmonate (MeJA) (Sarkar et al. 2017).

Plant defensins exist in various plant tissues, including tubers, leaves, flowers, and pods, but most have been found in seeds and roots. Moreover, defensins have broadspectrum antifungal and antibacterial activities (Franco et al. 2006). The defensin protein AFP2 in Raphanus sativus has in vitro antibacterial activities against Fusarium graminearum and Rhizoctonia solani, and wheat plants overexpressing RSAFP2 have increased resistance to both pathogens ( $\mathrm{Li}$ et al. 2011). The recombinant winter wheat defensin TAD1 protein inhibits the mycelial growth of Typhula ishikariensis in vitro, while transgenic wheat overexpressing TAD1 shows resistance to T. ishikariensis and $F$. graminearum (Sasaki et al. 2016). In addition, class I (NaD2) and class II ( $\mathrm{NaDl})$ defensins from Nepenthes alata flowers inhibit the germination of urediniospores and confer resistance to crown rust in oat seedlings when applied as a foliar spray (Dracatos et al. 2014). The Medicago sativa seed defensin MsDefl inhibits the growth of $F$. graminearum in vitro, and its antifungal activity was significantly reduced in the presence of $\mathrm{Ca}^{2+}$ (Spelbrink et al. 2004).

Panax notoginseng is a traditional Chinese medicinal herb with various biological activities, such as reducing swelling, relieving pain, promoting blood circulation, and alleviating blood stasis (Zhang et al. 2016). P. notoginseng is mainly distributed in southwestern China, and Wenshan Prefecture of Yunnan Province is the main producing area. During the artificial cultivation of $P$. notoginseng, root rot, black spot, and other fungal diseases severely affect the production and quality of the medicinal materials generated from P. notoginseng. Among them, root rot, which is mainly caused by Fusarium solani, is the most serious disease (Miao et al. 2006, Fan et al. 2016). The pretreatment of $P$. notoginseng with $100 \mu \mathrm{M}$ MeJA significantly increased its resistance to $F$. solani in our previous study. Therefore, the transcriptome sequencing of $P$. notoginseng roots pretreated with MeJA and then inoculated with $F$. solani was performed [the transcriptome data were submitted to the NCBI (SRA and TSA; accession numbers SRP149049 and GGPQ00000000, respectively). A differentially expressed gene encoding a DEFL was induced by the MeJA treatment and responded to $F$. solani infection. Here, to gain a deeper understanding of the defensin's function in the molecular interaction between $P$. notoginseng and $F$. solani, the full-length cDNA sequence of the defensin gene, named PnDEFL1 (GenBank accession number MK238492), was obtained. In addition, the expression profile, prokaryotic expression, and subcellular localization of PnDEFL1 were analyzed. PnDEFL1 was also overexpressed in tobacco to further investigate its function in response to $F$. solani infection.

\section{Materials and methods}

Plants, treatments, and inoculation: The Panax notoginseng (Burk) F.H. Chen seedlings used in the gene cloning and gene expression analysis were planted and grown in a greenhouse at a temperature of $25{ }^{\circ} \mathrm{C}$ and a relative humidity of $60 \%$ ). Sterile seedlings of tobacco (Nicotiana tabacum L. cv Xanthi) were cultured in a climatic cabinet at a temperature of $25{ }^{\circ} \mathrm{C}$ and a $16-\mathrm{h}$ photoperiod, and used for genetic transformation. Mycelia of F. solani, Botrosphaeria dothidea, Fusarium oxysporum, and Sclerotinia sclerotiorum that had been preserved at $-4{ }^{\circ} \mathrm{C}$ were activated on potato dextrose agar (PDA) media before using in a constant temperature incubator $\left(28^{\circ} \mathrm{C}\right)$.

For the chemical treatments, the concentrations of four stress-related signalling molecules, SA, MeJA, ethephon, and $\mathrm{H}_{2} \mathrm{O}_{2}$, were determined by referring to Lilium regale and Juglans sigillata treatment methods (Zhang et al. 2017, Liu et al. 2018), and then modified based on growth observations. The $P$. notoginseng seedlings were grown under normal conditions for 4 weeks after independent treatments with SA $(200 \mu \mathrm{M})$, JA $(100 \mu \mathrm{M})$, ethephon (1 mM), and $\mathrm{H}_{2} \mathrm{O}_{2}(1 \mathrm{mM})$. The roots of healthy oneyear-old $P$. notoginseng seedlings were wounded with scissors, and then the wounded $P$. notoginseng seedlings were treated with signalling molecule solutions using the dip-root method for $30 \mathrm{~min}$. The roots were collected after at $4,12,24,48$, and $72 \mathrm{~h}$ after treatment. For the fungal inoculations, the wounded roots of healthy one-year-old $P$. notoginseng seedlings were soaked with a solution of MeJA (100 $\mu \mathrm{M}$, treatment group) or sterile water (control group) for $30 \mathrm{~min}$. At $24-\mathrm{h}$ after the pretreatment, the two 
groups of $P$. notoginseng seedlings were inoculated with fresh spore suspensions of $F$. solani $\left(10^{6}\right.$ spores $\left.\mathrm{dm}^{-3}\right)$ for $30 \mathrm{~min}$. The roots were collected at $4,12,24,48$, and $72 \mathrm{~h}$ post-inoculation (hpi). Moreover, to eliminate individual differences, the root samples used for each chemical treatment and inoculation were collected from three different healthy $P$. notoginseng plants, and the roots were mixed and thoroughly ground in liquid nitrogen before RNA extraction.

Rapid-amplification of cDNA ends (RACE) and bioinformatics analysis: The full-length cDNA was amplified using a SMART RACE cDNA amplification kit (Clontech, Palo Alto, USA), and the gene-specific primers (Table 1 Suppl.) were designed based on the unigene sequence of DEFL using Primer Premier 5 software. The mRNA was isolated from $100 \mu \mathrm{g}$ of $P$. notoginseng total RNA using a NucleoTrap ${ }^{\circledR}$ mRNA midi kit (MachereyNagel, Düren, Germany), and the cDNA synthesis, as well as RACE-PCR, were performed according to the manufacturer's protocols (Clontech). The PCR product was cloned into the pGEM-T easy vector (Promega, Madison, USA) and transformed into Escherichia coli DH5 $\alpha$ competent cells. The recombinant plasmid was selected for bidirectional sequencing. The overlap and assembly of unigene sequence and RACE products, as well as the identification of open reading frame (ORF), were performed using the $N C B I$ website (https://www.ncbi.nlm. nih.gov/). The other bioinformatics analysis methods used were previously described in Li et al. (2014).

Quantitative reverse transcription-PCR (RT-qPCR) was used to analyze the expression patterns of PnDEFL1 in $P$. notoginseng roots during $F$. solani infection and after MeJA, ETH, $\mathrm{H}_{2} \mathrm{O}_{2}$, and SA treatments. The P. notoginseng actin 2 gene (PnACT2, GenBank accession number KF815706.1) was used as the internal control for the standardization of different RNA samples. Gene-specific primers for the PnDEFL1 and PnACT2 were designed based on the corresponding cDNA sequences using Primer Premier 5 software (Table 1). The detailed RT-qPCR scheme was the same as described in Liu et al. (2013a). Relative gene expressions were calculated using the $2^{-\Delta \Delta C t}$ method (Livak and Schmittgen 2001). The expression of the PnDEFL1 gene in wounded $P$. notoginseng roots without treatment or inoculation was considered as the control, and its relative expression value was designated as one. The RT-qPCR assay was carried out with three replicates.

Subcellular localization of PnDEFL1: The pGEM-T easy-PnDEFL1 and pBIN m-gfp5-ER (Zhang et al. 2017) plasmids were digested with the restriction enzymes $N d e$ I and SmaI, and then ligated with T4 DNA ligase (Promega). The PnDEFL1 gene was fused with the green fluorescent protein $(G F P)$ gene present in the pBIN m-gfp5-ER vector under the control of the CaMV $35 \mathrm{~S}$ promoter to generate the PnDEFL1-GFP fusion protein. The pBIN m-gfp5-ER-PnDEFL1 recombinant plasmid was then transformed into the Agrobacterium tumefaciens strain
EHA105 and plated onto Lurie brot (LB) solid medium containing $50 \mathrm{mg} \mathrm{dm}^{-3}$ kanamycin and $20 \mathrm{mg} \mathrm{dm}^{-3}$ rifampicin. The $A$. tumefaciens positive clones harbouring recombinant plasmids were confirmed by PCR and used for transient expression in onion (Allium cepa) epidermal cells. The detailed procedure of the A. tumefaciensmediated genetic transformation and GFP observations with laser confocal microscopy (A1R, Nikon, Tokyo, Japan) were previously described in Liu et al. (2018).

Expression and purification of recombinant PnDEFL1 protein: The pGEM-T easy-PnDEFL1 plasmids were digested with NdeI and SmaI, and then the PnDEFL1 ORF, without the stop codon, was subcloned into the C-terminus of the pET-32a vector to generate a $6 \times$ His-tagged fusion protein in Escherichia coli. E. coli strain BL21 (DE3) containing the pET-32a-PnDEFL1 plasmid was grown in liquid LB medium supplemented with ampicillin $\left(50 \mathrm{mg} \mathrm{dm}{ }^{-3}\right)$ in a shaker at $37^{\circ} \mathrm{C}$. When the absorbance at $600 \mathrm{~nm}$ reached $0.6-0.8$, isopropyl-1-thio- $\beta$-D-gactopyranoside (IPTG, $1 \mathrm{mM}$ final concentration) was added to the LB medium, and the E. coli cells were further cultured in a shaker at $25{ }^{\circ} \mathrm{C}$. The $E$. coli cells were collected after induction for $8 \mathrm{~h}$. The cells were first lysed by lysozyme, and then the supernatant and the precipitate were separately sampled. The recombinant PnDEFL1 protein was purified from the supernatant using a $\mathrm{Ni}-\mathrm{NTA}$ column according to manufacturer's instructions (Sangon, Shanghai, China).

Antifungal assay of recombinant PnDEFL1 protein: Four fungi, F. solani, F. oxysporum, B. dothidea, and $S$. sclerotiorum were activated on PDA solid medium at $28^{\circ} \mathrm{C}$. The activated mycelia were inoculated on the center of the PDA solid medium, and when the radii of the fungal colonies were $\sim 2 \mathrm{~cm}$, sterilized filter-paper discs $(0.6 \mathrm{~mm}$ in diameter) were placed uniformly around the fungal colonies. Then, 5,10 , and $15 \mu \mathrm{g}$ of purified recombinant PnDEFL1 protein were added independently to the filter paper. In addition, $0.01 \mathrm{~cm}^{3}$ of $\mathrm{ddH}_{2} \mathrm{O}$ and buffer were added as blank controls. All the culture dishes were placed in an incubator at $28^{\circ} \mathrm{C}$ for $3 \mathrm{~d}$, and then, the antifungal activity of the PnDEFL1 recombinant protein against the four fungi was recorded using a digital camera (Canon, Tokyo, Japan). The zones of growth inhibition around the disks were calculated using Adobe Photoshop v. 7.0, and three replications of the antifungal assay were performed.

Overexpression vector construction and tobacco genetic transformation: The plant vector for overexpressing PnDEFL1 was constructed using the pCAMBIA2300s vector (Liu et al. 2013b). The ORF of PnDEFL1 was obtained by digesting pGEM-T easy-PnDEFL1 with $B a m H I$ and $E c o R I$ and was then ligated into the digested pCAMBIA2300s vector using the same restriction enzymes. The ligation product was transformed into E. coli DH5a competent cells, and positive clones harbouring the recombinant plasmid pCAMBIA2300sPnDEFL1 were selected using PCR. The recombinant plasmid pCAMBIA2300S-PnDEFL1 was further 
transformed into the competent cells of A. tumefaciens strain LBA4404 using the freeze-thaw method (Holsters et al. 1978). The tobacco (N. tabacum L. cv. Xanthi) leaf discs were transformed using the LBA4404 bacterial suspension containing the recombinant vector pCAMBIA2300S-PnDEFL1 (Horsch et al. 1985).

Transgenic tobacco analysis: The tobacco seedlings were regenerated through tissue culture, and the positive transgenic tobacco plants were screened using PCR. The genomic DNA of T0 generation of tobacco plants was the template for PCR with PnDEFL1-specific primers (Table 1), and the wild type (WT) genomic DNA was the negative control. Moreover, the positive PnDEFL1 transgenic tobacco plants were cultured in a greenhouse to develop T2 generation tobacco lines, which were confirmed using PCR with PnDEFL1-specific primers. The expression of PnDEFL1 in the T2 generation transgenic tobacco was analyzed using RT-qPCR. The tobacco actin gene (AB158612.1) was used as the internal control for the standardization of different RNA samples, and WT tobacco was used as the negative control. The transgenic tobacco line with the highest $\mathrm{Ct}$ value was taken as a positive control, and the value of PnDEFL1 expression in the positive control was designated as one.

The sterile T2 tobacco lines, as well as the WT plants, were cultured in a half strength Murashige and Skoog medium for 2 months in an artificial climatic incubator (25 ${ }^{\circ} \mathrm{C}$, a 16 -h photoperiod), and several tobacco lines with relatively high $P n D E F L 1$ transcription were selected to estimate the resistance to $F$. solani infection. The fully expanded tobacco leaves were partially wounded and inoculated with $0.02 \mathrm{~cm}^{3}$ of $F$. solani spore suspension $\left(10^{6}\right.$ spores $\left.\mathrm{cm}^{-3}\right)$. The infected leaves were placed on a damp filter paper and placed in a light incubator. One week after inoculation, the infected leaves were collected and lesions caused by $F$. solani infection were recorded. In addition, the wounded roots of sterile transgenic and WT tobacco were immersed in the $F$. solani spore suspension $\left(2 \times 10^{6}\right.$ spore $\left.\mathrm{cm}^{-3}\right)$ for $30 \mathrm{~min}$ and then transferred into a half strength Murashige and Skoog culture medium. The tobacco symptoms were recorded at one week after inoculation.

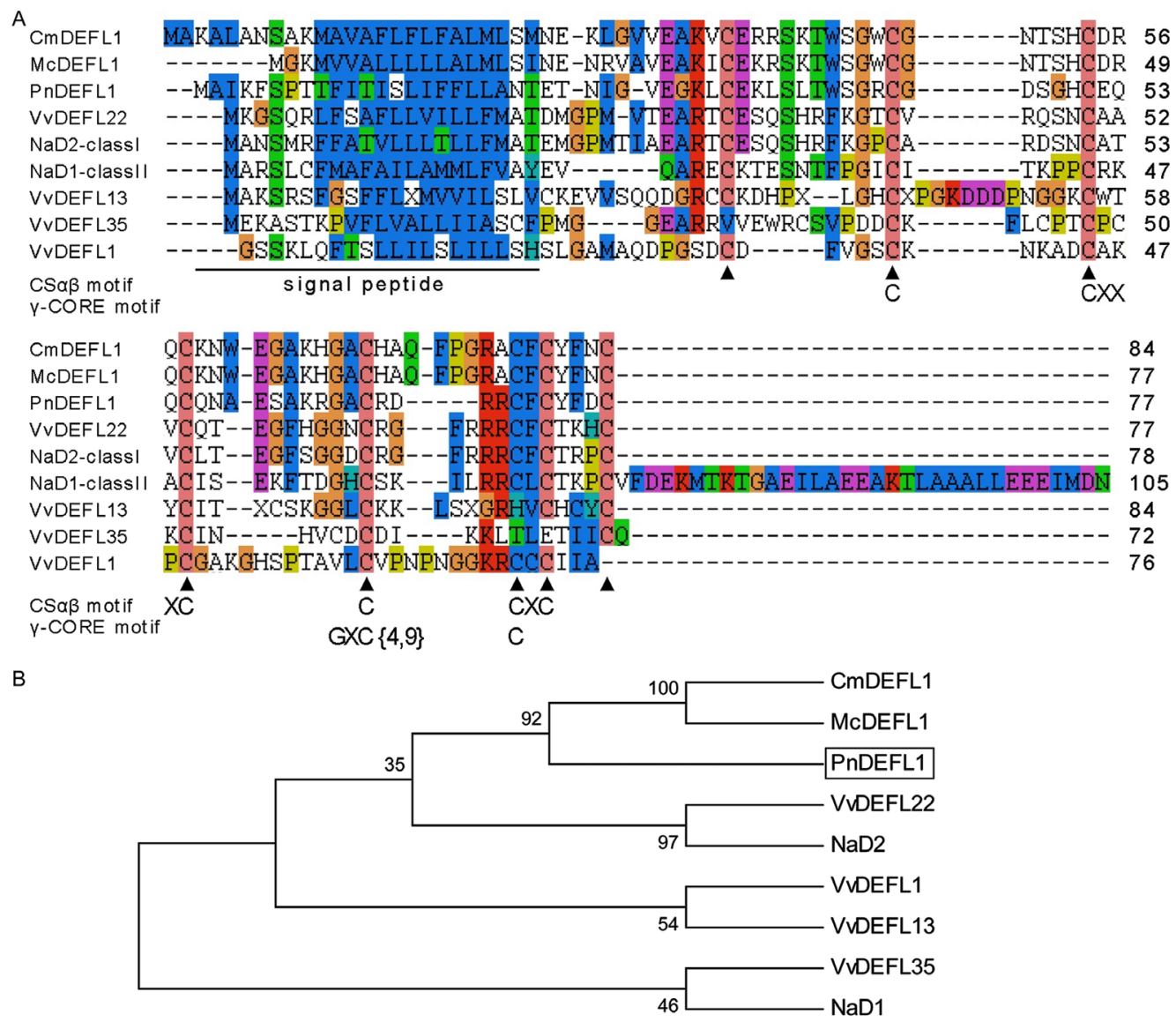

Fig. 1. A multiple alignment and phylogenetic tree of the amino acid sequences of PnDEFL1 and several known defensins or defensin like proteins (DEFLs). The multiple alignment was completed using ClustalX $(A)$. The signal peptide is labeled with a horizontal line, and the highly conserved cysteine residues are labeled with triangles. Defensins included in the alignment are two Nicotiana alata defensins (NaD1 and NaD2) (Dracatos et al. 2014), four DEFLs from Vitis vinifera (VvDEFL1, VvDEFL13, VvDEFL22, and VvDEFL35) (Giacomelli et al. 2012), Cucumis melo CmDEFL1 (XP_008454663.1), and Momordica charantia McDEFL1 (XP_022139709.1), respectively. The phylogenetic tree was constructed using the Mega 6 software with the neighbour-joining method (B). The scale bar equals the distance of 10 changes per 100 amino acid positions, and the numbers on each node are bootstrap values of 1000 replicates. 
Statistical analyses: The above experiments were repeated three times. The relative expressions of PnDEFL1 in $P$. notoginseng and T2 tobacco lines and the zones of fungal growth inhibition are shown as means and standard deviations. The Student's $t$-test was performed using the SPSS software to reveal statistical differences between the treatments or inoculations and controls.

\section{Results}

In this study, a DEFL gene PnDEFL1 was isolated from $P$. notoginseng. The 5'and 3' RACE PCR products were $301 \mathrm{bp}$ and $487 \mathrm{bp}$, respectively, and the overlap and assembly of unigene sequence and RACE products generated a cDNA of $702 \mathrm{bp}$, in which an ORF of 234 bp was identified. Moreover, the ORF encodes a complete protein sequence of 77 amino acid residues that is highly homologous to plant DEFLs. The calculated molecular mass of the deduced protein PnDEFL1 is $8.63 \mathrm{kDa}$, with a theoretical pI of 7.51. At the nucleotide level, the PnDEFL1 gene shared a $74 \%$ identity with the Helianthus annuus DEFL19 gene (XM_022160565.1). At the protein level, the deduced protein sequence of
PnDEFL1 shared a $58 \%$ identity with DEFL1 of Cucumis melo (XP_008454663.1) and a $54 \%$ identity with Momordica charantia DEFL1 (XP_022139709.1).

The class I and II defensins derived from the flowers of N. alata (Dracatos et al. 2014), four kinds of DEFLs from $V$. vinifera (Giacomelli et al. 2012), and two homologous sequences (CmDEFL1 and McDEFL1) from the protein $B L A S T$ analysis were chosen for the multiple sequence alignment and phylogenetic tree analysis. PnDEFL1 had a predicted signal peptide positioned at its N-terminus like other defensins, and in addition, the hallmarks of defensins, the $\mathrm{CS} \alpha \beta$ and $\gamma$-core motifs, were present (Fig. 1A). The majority of the plant defensins or DEFLs contain eight highly conserved cysteine residues. For the phylogenetic tree, VvDEFL35 and NaD1 were grouped at the bottom of the tree, while PnDEFL1, the other DEFLs, and NaD2 clustered into another group (Fig. 1B). PnDEFL1 was more homologous with the class I defensin (NaD2) than the class II defensin $(\mathrm{NaD} 1)$.

The transcriptions of PnDEFL1 in P. notoginseng roots after independent treatments with four different signalling molecules were analyzed using RT-qPCR. The expression of PnDEFL1 was rapidly induced by MeJA, ETH, $\mathrm{H}_{2} \mathrm{O}_{2}$, and SA treatments (Fig. $2 A-D$ ), but

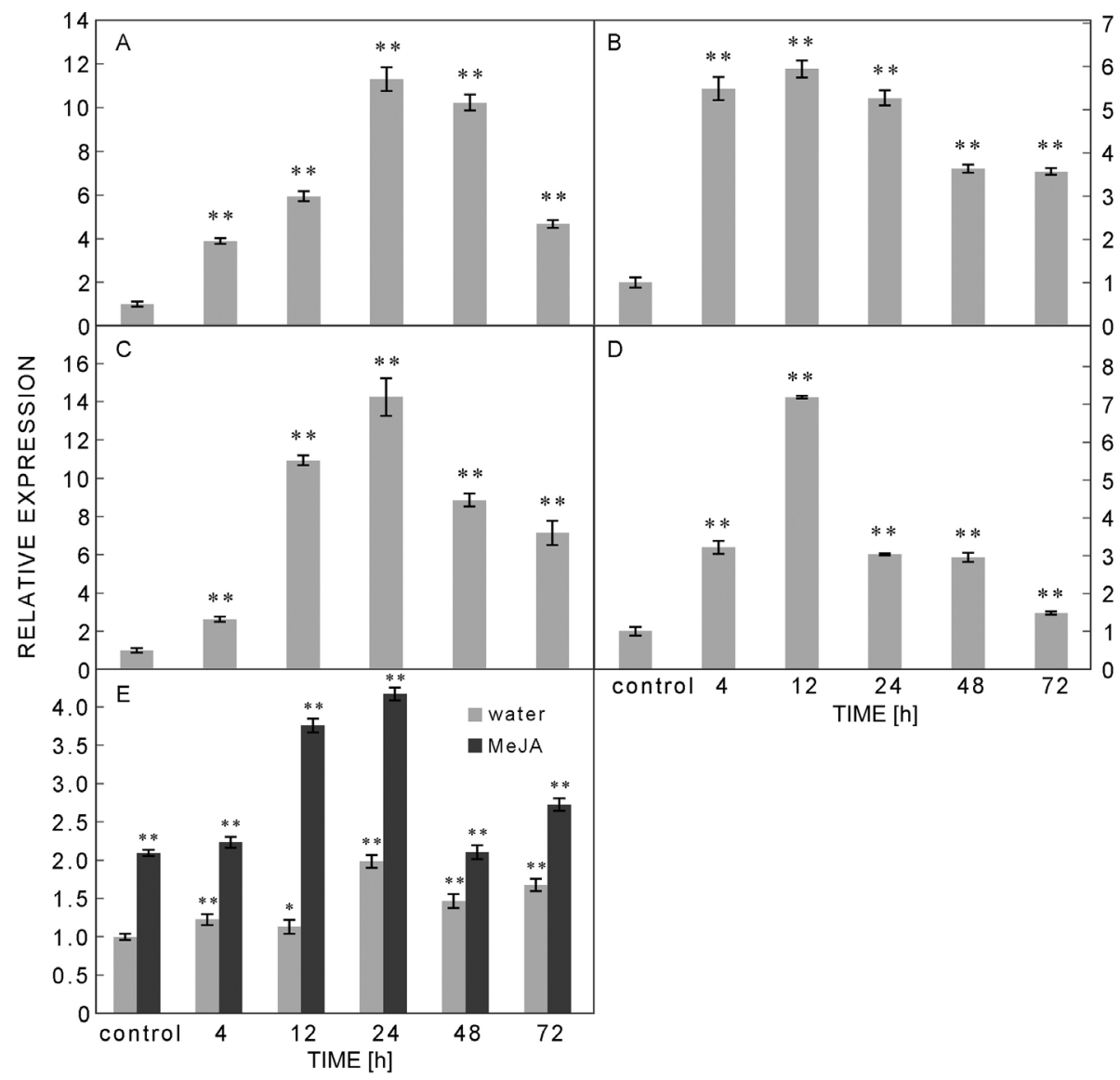

Fig. 2. The expression profiles of PnDEFL1 in roots of Panax notoginseng. The PnDEFL1 expressions after treatments with four signaling molecules including methyl jasmonate $(A)$, salicylic acid $(B)$, ethylene $(C)$, and $\mathrm{H}_{2} \mathrm{O}_{2}(D)$, as well as after inoculation with Fusarium solani $(E)$ were analyzed by reverse transcription quantitative PCR. Means \pm SEs of three biological replicates, and the statistical differences between the treatments and the controls were analyzed by the Student's $t$ test $\left(*^{*} P<0.05,{ }^{* *}-P<0.01\right)$. 
the expressions of PnDEFL1 were different. The greatest expression occurred after the ETH treatment, followed by MeJA, SA, and $\mathrm{H}_{2} \mathrm{O}_{2}$ treatments. Moreover, the highest relative expressions of PnDEFL1 were reached at 24, 12, 24, and $12 \mathrm{~h}$ for the MeJA, SA, ETH, and $\mathrm{H}_{2} \mathrm{O}_{2}$ treatments, respectively. Compared with the sterile water treatment, the PnDEFL1 expression was highly induced ( $\sim 12$ times) by the exogenous application of MeJA at $24 \mathrm{~h}$. After $F$. solani inoculation, the PnDEFL1 expression in the sterile water pre-treatment group rapidly increased; however, the PnDEFL1 expression in MeJA pre-treatment group were even greater than that in the water pre-treatment group (Fig. 2E). In particular, at $24 \mathrm{~h}$, the relative expression value in the MeJA pre-treatment group was $\sim 2.1$ times greater than in the water pre-treatment group (Fig. 2E). Furthermore, the overall PnDEFL1 expressions were greater in the MeJA group than in the water group until $72 \mathrm{~h}$.

Onion epidermal cells were independently infected with $A$. tumefaciens carrying the pBIN m-gfp5-ERPnDEFL1 and the empty pBIN m-gfp5-ER vector (positive control), and the green fluorescence signal was observed using a laser confocal microscope (Fig. 3). In the positive control, the green fluorescence was distributed throughout the onion cells harbouring the empty pBIN m-gfp5-ER vector. The green fluorescence of onion cells harbouring the pBIN m-gfp5-ER-PnDEFL1 vector was specifically distributed in the cell wall, indicating that PnDEFL1 is an extracellular protein that localized in the plant cell wall.

The PnDEFL1 protein without a signal peptide was expressed in E. coli, and the size of the PnDEFL1 recombinant protein was approximately $25 \mathrm{kDa}$, which was consistent with the predicted size of the histidine-tagged fusion protein (Fig. 4A). The PnDEFL1 fusion protein was purified by nickel affinity chromatography. The antifungal assay showed that the PnDEFL1 recombinant protein inhibited the mycelial growth of $F$. solani, $F$. oxysporum, $B$. dothidea, and $S$. sclerotinia in vitro (Fig. $4 B-E$ ). In addition, greater amount of the PnDEFL1 protein exhibited more pronounced inhibitory effects on the four pathogenic fungi growth (Fig. 4F).

The positive PnDEFL1 transgenic tobacco plants were grown in a greenhouse until T2 generations were obtained. No significant differences were observed between the PnDEFL1 transgenic and WT tobacco in growth and morphogenesis. The expressions of PnDEFL1 in T2 generation transgenic tobacco leaves were detected using RT-qPCR. The PnDEFL1 transcripts only accumulated in nine transgenic tobacco lines (D-2, -4, -5, -7, -12, -16, -17, -18 , and -23$)$, but not in the WT, and the expressions in these transgenic lines differed (Fig. 5A). The expressions in the transgenic tobacco lines D-2, $-7,-18$, and -23 were relatively low, with values below 20; however, the expressions in the lines D-4, $-5,-12,-16$, and -17 were much greater than in other lines.

The PnDEFL1 transgenic and WT tobacco leaves were inoculated with $F$. solani spore suspensions. After $7 \mathrm{~d}$ of incubation, the leaves of WT tobacco showed extremely severe chlorosis and decay over a large area; however, the PnDEFL1 transgenic tobacco leaves showed almost no symptoms (Fig. 5B). In addition, the $F$. solani spore suspension was used to inoculate the wounded roots of PnDEFL1 transgenic and WT tobacco. After being cultured in a climatic cabinet for $7 \mathrm{~d}$ after inoculation, the WT tobacco roots were seriously rotted and blackened, and the leaves were withered and yellowed (Fig. 5C). On the contrary, the roots of PnDEFLl transgenic tobacco exhibited limited blackening, and the plants showed
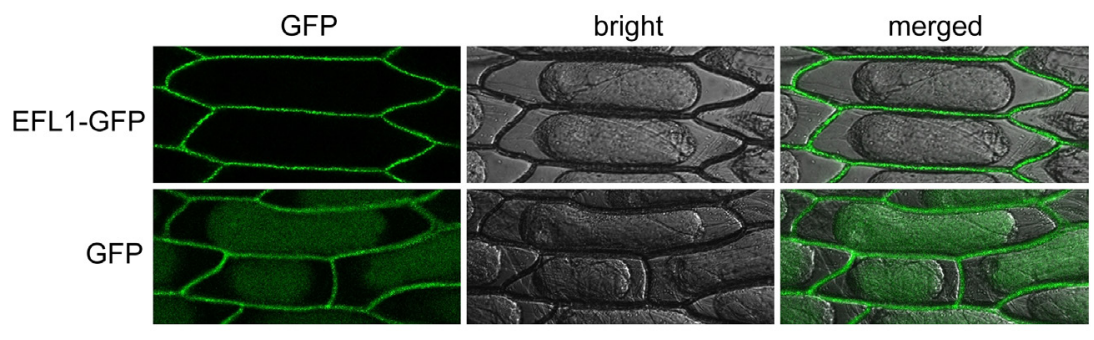

Fig. 3. Subcellular localization analysis indicates that the PnDEFL1 protein was localized in the cell wall. The PnDEFL1-GFP fusion gene and greenfluorescent protein (GFP, control) were transiently expressed in onion epidermal cells mediated by Agrobacterium tumefaciens. The onion epidermal tissue was treated with a $30 \%$ sucrose solution to induce plasmolysis, and then a confocal laser microscopy was used to display GFP (green fluorescence), bright field (bright), and combination (merged) views, respectively.

Fig. 4. (See the next page.) The expression of the recombinant PnDEFL1 protein in Escherichia coli BL21 (DE3) was analyzed through SDS-PAGE (A). M - protein marker; 1 - total protein of $E$. coli BL21 (DE3) containing pET-32(a) empty vector induced by isopropyl-1thio- $\beta$-D-gac-topyranoside (IPTG, $1 \mathrm{mM}$ ) for $7 \mathrm{~h} ; 2$ - total protein of $E$. coli BL21 (DE3) containing pET-32(a)-PnDEFL1 recombinant vector without induction; 3, 4, and 5 - total proteins of E. coli BL21 (DE3) containing pET-32(a)-PnDEFL1 cultured for 3, 5, and $7 \mathrm{~h}$ with the induction by $0.5 \mathrm{mM}$ IPTG, respectively; $6,7,8$, and 9 - total protein of $E$. coli BL21 (DE3) containing pET-32(a)- PnDEFL1 cultured for $3,5,7$, and $8 \mathrm{~h}$ with the induction by $1 \mathrm{mM}$ IPTG, respectively. The SDS-PAGE analysis of the His-tagged recombinant PnDEFL1 protein purified from $E$. coli $(B)$. M - protein marker; 1 - total protein of $E$. coli BL21 (DE3) containing pET-32(a)-PnDEFL1 recombinant vector without induction; 2, 3, 4, 5, 6, and 7 - purified PnDEFL1 protein eluted with 50, 100, 150, 200, 250, and 300 mM imidazole buffer, respectively. The recombinant PnDEFL1 protein has an evident antifungal activity to $F$. solani $(C), F$. oxysporum $(D)$, $S$. sclerotiorum $(E)$, and $B$. dothidea $(F)$. In addition, the fungal growth inhibition analysis shows the antifungal activities increased with the increase of recombinant PnDEFL1 protein $(G)$. The experiment was repeated three times to obtain an average size of fungal growth inhibition, and the statistical differences were analyzed by the Duncan's multiple range test. 

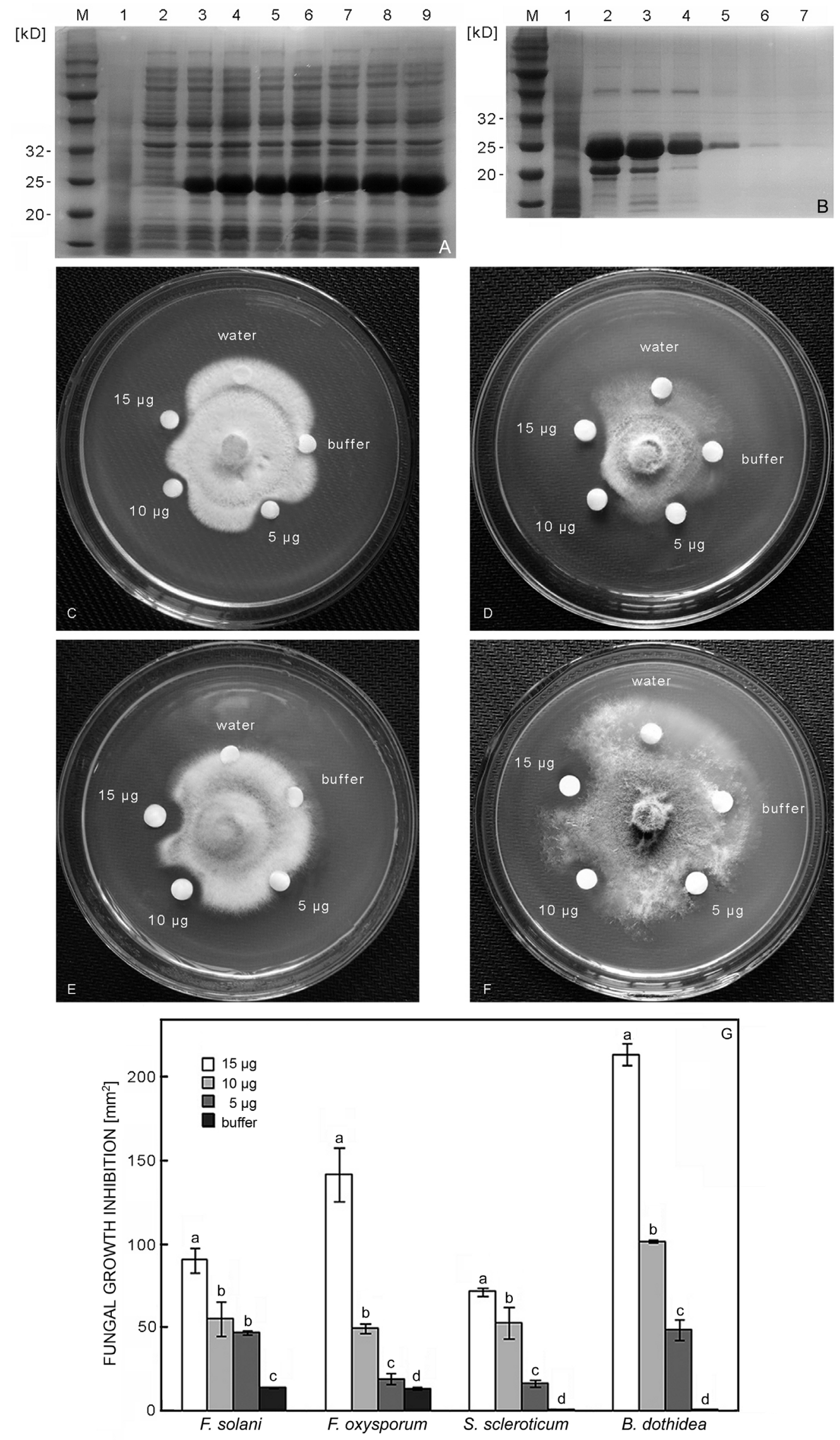
almost healthy growth state (Fig. $5 C$ ). Thus, the PnDEFL1 transgenic tobacco lines were much more resistant to $F$. solani infection than the WT tobacco, which suggested that the overexpression of PnDEFL1 in tobacco conferred a strong resistance to $F$. solani.

\section{Discussion}

Plant defensins belong to the plant AMPs (Broekaert et al. 1997), which are important parts of the plant innate immune system. They have an inhibitory effect on pathogenic microorganisms, such as bacteria and fungi, and play an important role in plant defense responses (Zasloff 2002). In the present study, a novel defensin-like protein gene PnDEFL1 was investigated and isolated from $P$. notoginseng. The full-length cDNA of PnDEFL1 is 702 bp and contains an ORF of $234 \mathrm{bp}$. The sequence analysis included several defensins and DEFLs from different plant species and showed that DEFLs have a diverse primary sequence. However, DEFLs contain the highly conserved structural features that are hallmarks of defensins, such as the $\operatorname{CS} \alpha \beta$ and $\gamma$-core motifs, and a specified number of cysteine residues. Previous genomic studies indicated that plant DEFLs and defensins constitute a supergene family, and the DEFLs are real defensins (Graham et al. 2004, Silverstein et al. 2005).

The bioinformatics analysis indicated that there is an N-terminal signal peptide predicted in the PnDEFL1 protein, and its subcellular localization was investigated using its fused expression with GFP. Although the green fluorescence of the empty vector with GFP was distributed throughout the cell wall and protoplasm, the fluorescence signal of the fusion gene PnDEFL1-GFP was detected only in the cell wall, which indicated that PnDEFL1 is localized in the cell wall. Like PnDEFL1, some other defensins and DEFLs are extracellular proteins. The two rice defensin genes $O_{s} D E F 7$ and $O s D E F 8$ have been fused with $G F P$, and the fusion proteins localized in the extracellular compartment (Weerawanich et al. 2018). In addition, the $V$. vinifera defensin antibacterial peptide Vv-AMP1 fused with GFP accumulated also in the extracellular region (De Beer and Vivier 2008). However, N. alata NaD1 has been immunolocalized in vacuoles (Lay et al. 2003). The A. halleri defensin AhPDF 1.1 remained in the intracellular rather than the extracellular compartment
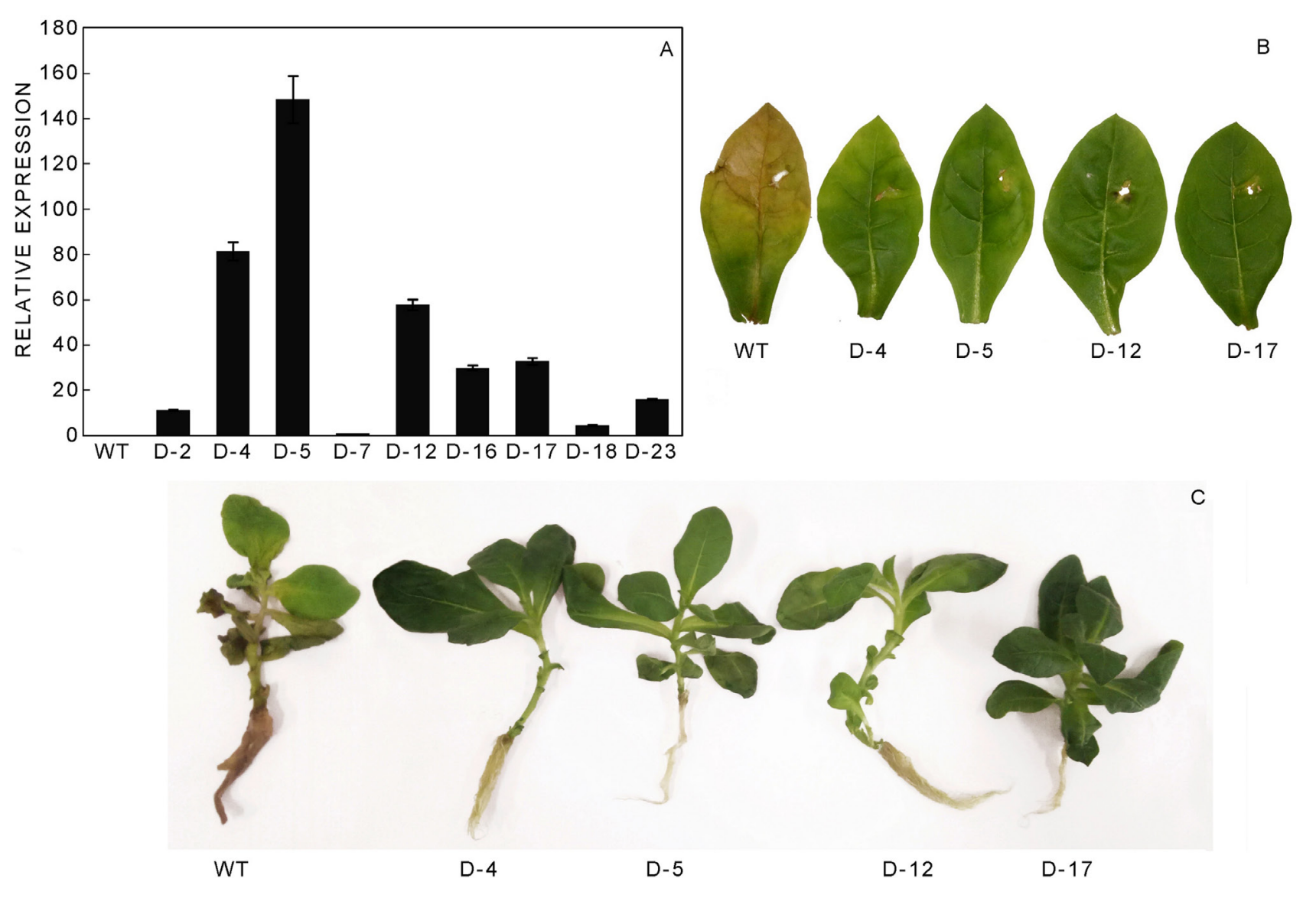

Fig. 5. The PnDEFL1 expressions and resistance analyses of T2 generation PnDEFL1 transgenic tobacco. The PnDEFL1 expressions in the T2 generation of transgenic tobacco were detected by reverse transcription quantitative PCR, and the PnDEFL1 transcripts accumulated in all the transgenic tobacco plants but not in the wild type (WT) (A). The D-2, $-4,-5,-7,-12,-16,-17,-18$, and -23 are $\mathrm{T} 2$ generation transgenic tobacco lines. Leaf-inoculation-assay revealed the enhanced resistance of PnDEFL1 transgenic tobacco lines against Fusarium solani $(B)$. Root-inoculation-assay revealed the enhanced resistance of PnDEFL1 transgenic tobacco lines against Fusarium solani $(C)$. 
on the way to the lytic vacuole (Oomen et al. 2011). The different localizations of defensins in plant cells suggest that defensins are involved in multiple physiological processes.

Plant defensins have been isolated from a variety of plants and expressed in different organs and tissues. Plant defensins are induced as a part of the systemic defense response during pathogenic attack and environmental stresses, and defensin genes respond to some signalling molecules, including MeJA, ETH, and SA. After inoculation with $F$. oxysporum, many DEFLs show enhanced expressions in induced resistancedisplaying wheat seedlings (Odintsova et al. 2019). The OsDEF expressions are highly induced by Aspergillus oryzae infection (Weerawanich et al. 2018). Compared with uninoculated seedlings, defensin PDF1.2b was significantly up-regulated in the $A$. thaliana leaves after inoculation with Alternaria brassicicola or Pseudomonas syringae (Tesfaye et al. 2013). Moreover, AtPDF1 responds to $B$. cinerea through the activation of the ETH/ JA signalling pathway (Nguyen et al. 2014). In addition, the expression of a defensin gene $J 1-1$ in pepper was induced by Colletotrichum gloeosporioides infection as well as an exogenous MeJA treatment (Seo et al. 2014). In this study, DEFL1 from $P$. notoginseng was induced by applications of MeJA, SA, ETH, or $\mathrm{H}_{2} \mathrm{O}_{2}$ (Fig. 2A-D). In addition, $P n D E F L 1$ 's expression was further up-regulated in the MeJA pre-treated $P$. notoginseng during the response to $F$. solani infection, with a maximum expression at 24 hpi (Fig. 2E). Thus, PnDEFL1 is involved in the defense response of $P$. notoginseng to $F$. solani, which may be mediated by several signalling pathways, including JA.

Multicellular organisms produce small cysteinerich AMPs as an innate defense against a broad range of pathogens. The plant defensins and DEFLs are cysteinerich AMPs. Because of a large number of cysteines, it may be difficult to express defensin peptides at high activity in vitro; however, their antimicrobial activities have been successfully obtained using heterologous expression systems. Four recombinant VvDEFLs purified from $E$. coli inhibited the conidial germination and mycelial growth of B. cinerea (Giacomelli et al. 2012). The Phaseolus vulgaris defensin protein PvD1 was expressed in E. coli, and the purified PvD1 inhibited the growth of Candida albicans (Mello et al. 2014). Lacerda et al. (2016) expressed the recombinant Pisum sativum defensin Drr230a in Saccharomyces cerevisiae, and the recombinant protein Drr230a showed in vitro activities against the fungal growth and spore germination of $C$. gossypii var. cephalosporioides. The pepper defensin protein J1-1 inhibited the mycelial growth of $F$. oxysporum and $B$. cinerea, and the recombinant $J 1-1 /$ GST fusion protein significantly inhibited the growth of $C$. gloeosporioides (Seo et al. 2014). In this study, the recombinant protein PnDEFL1 purified from E. coli significantly inhibited the mycelial growth of F. solani, F. oxysporum, B. dothidea, and S. sclerotinia (Fig. 4). F. solani and F. oxysporum are both important agents that cause root rot disease in $P$. notoginseng, and the antifungal activity of PnDEFL1 clearly indicated that PnDEFL1 plays a vital role in the defense against root rot disease in P. notoginseng.

In addition, the biological functions of plant defensins as natural antibiotics were confirmed using reverse genetics. The overexpression of AtPDF1.1 in $A$. thaliana reduced infection by the non-parasitic pathogen Cercospora beticola (De Coninck et al. 2010). Petunia floral defensins have an in vitro antifungal activity against $F$. oxysporum, and transgenic bananas overexpressing PhDefl and PhDef2 increase resistance to F. oxysporum (Ghag et al. 2012). The plant defensin NaDl of $N$. alata has been overexpressed in cotton plants, compared with nontransgenic cv. Coker315, and the transgenic line D1 has enhanced the resistance to $F$. oxysporum and Verticillium dahliae (Gaspar et al. 2014). The constitutive expression of $J 1-1$ endowed transgenic pepper plants with a strong resistance to the anthracnose fungus (Seo et al. 2014). Moreover, the expression of Def4.2 from M. truncatula in transgenic wheat confers resistance to leaf rust caused by the biotrophic basidiomycete fungus Puccinia triticina (Kaur et al. 2017). Here, the PnDEFL1 transgenic tobacco exhibited a much greater resistance to $F$. solani infection than the WT (Fig. 5B,C), which is conferred by the overexpression of PnDEFL1.

In conclusion, PnDEFL1, a defensin like protein gene, was isolated from $P$. notoginseng and characterized. PnDEFL1 expression was induced by $F$. solani infection and exogenous signalling molecules. In addition, the recombinant PnDEFL1 protein had significant antifungal activities against four pathogenic fungi. The overexpression of PnDEFL1 also conferred a high resistance to F. solani infection. PnDEFL1 appears to be a key component of the defense system in $P$. notoginseng during responses to root rot disease.

\section{References}

Bahramnejad, B., Erickson, L.R., Atnaseo, C., Goodwin, P.H.: Differential expression of eight defensin genes of $N$. benthamiana following biotic stress, wounding, ethylene, and benzothiadiazole treatments. -Plant Cell Rep. 28: 703-717, 2009.

Broekaert, W.F., Cammue, B.P.A., Bolle, M.F.C.D., Thevissen, K., Samblanx, G.W.D., Osborn, R.W., Nielson, K.: Antimicrobial peptides from plants. - Crit. Rev. Plant Sci. 16: 297-323, 1997.

Cui, J., Jiang, N., Meng, J., Hou, X.X., Yang, G.L., Luan, Y.S.: Identification and characterization of defensin genes conferring Phytophthora infestans resistance in tomato. Physiol. mol. Plant Pathol. 103: 28-35, 2018.

Colilla, F.J., Rocher, A., Mendez, E.: $\gamma$-Purothionins: amino acid sequence of two polypeptides of a new family of thionins from wheat endosperm. - FEBS Lett. 270: 191-194, 1990.

De Beer, A., Vivier, M.A.: Vv-AMP1, a ripening induced peptide from Vitis vinifera shows strong antifungal activity. - BMC Plant Biol. 8: 75, 2008.

De Coninck, B.M., Sels, J., Venmans, E., Thys, W., Goderis, I.J., Carron, D., Delauré, S.L., Cammue, B.P., De Bolle, M.F., Mathys, J.: Arabidopsis thaliana plant defensin AtPDF1.1 is involved in the plant response to biotic stress. - New Phytol. 187: 1075-1088, 2010.

De Souza, C.E., Silva Cardoso, M.H., Sousa, D.A., Viana, J.C., 
De Oliveira Jr., N.G., Miranda, V., Franco, O.L.: The use of versatile plant antimicrobial peptides in agribusiness and human health. - Peptides 55: 65-78, 2014.

Do, H.M., Lee, S.C., Jung, H.W., Sohn, K.H., Hwang, B.K.: Differential expression and in situ localization of a pepper defensin (CADEF1) gene in response to pathogen infection, abiotic elicitors and environmental stresses in Capsicum annuum. - Plant Sci. 166: 1297-1305, 2004.

Dracatos, P.M., Van der Weerden, N.L., Carroll, K.T., Johnson, E.D., Plummer, K.M., Anderson, M.A.: Inhibition of cereal rust fungi by both class I and II defensins derived from the flowers of Nicotiana alata. - Mol. Plant Pathol. 15: 67-79, 2014.

Fan, Z.Y., Miao, C.P., Qiao, X.G., Zheng, Y.K., Chen, H.H., Chen, Y.W., Xu, L.H., Zhao, L.X., Guan, H.L.: Diversity, distribution, and antagonistic activities of rhizobacteria of Panax notoginseng. - J. Ginseng Res. 40: 97-104, 2016.

Fant, F., Vranken, W., Broekaert, W., Borremans, F.: Determination of the three-dimensional solution structure of Raphanus sativus antifungal protein 1 by $1 \mathrm{H}$ NMR. - J. mol. Biol. 279: 257-270, 1998.

Franco, O.L., Murad, A.M., Leite, J.R., Mendes, P.A., Prates, M.V., Bloch, C. Jr.: Identification of a cowpea gamma-thionin with bactericidal activity. - FEBS J. 273: 3489-3497, 2006

Gachomo, E.W., Jimenez-Lopez, J.C., Kayodé, A.P., BabaMoussa, L., Kotchoni, S.O.: Structural characterization of plant defensin protein superfamily. - Mol. Biol. Rep. 39: 4461-4469, 2012.

Gaspar, Y.M., McKenna, J.A., McGinness, B.S., Hinch, J., Poon, S., Connelly, A.A., Anderson, M.A., Heath, R.L.: Field resistance to Fusarium oxysporum and Verticillium dahliae in transgenic cotton expressing the plant defensin $\mathrm{NaD1}$. - J. exp. Bot. 65: 1541-1550, 2014.

Ghag, S.B., Shekhawat, U.K., Ganapathi, T.R.: Petunia floral defensins with unique prodomains as novel candidates for development of Fusarium wilt resistance in transgenic banana plants. - PLoS ONE 7: e39557, 2012.

Giacomelli, L., Nanni, V., Lenzi, L., Zhuang, J., Dalla Serra, M., Banfield, M.J., Town, C.D., Silverstein, K.A., Baraldi, E., Moser, C.: Identification and characterization of the defensinlike gene family of grapevine. - Mol. Plant Microbe Interact. 25: 1118-1131, 2012.

Graham, M.A., Silverstein, K.A., Cannon, S.B., Van den Bosch, K.A.: Computational identification and characterization of novel genes from legumes. - Plant Physiol. 135: 1179-97, 2004.

Kaur, J., Fellers, J., Adholeya, A., Velivelli, S.L., El-Mounadi, K., Nersesian, N., Clemente, T., Shah, D.: Expression of apoplast-targeted plant defensin MtDef4.2 confers resistance to leaf rust pathogen Puccinia triticina but does not affect mycorrhizal symbiosis in transgenic wheat. - Transgenic Res. 26: 37-49, 2017.

Holsters, M., De Waele, D., Depicker, A., Messens, E., Van Montagu, M., Schell, J.: Transfection and transformation of Agrobacterium tumefaciens. - Mol. gen. Genet. 163: 181-187, 1978.

Horsch, R.B., Fry, J.E., Hoffmann, N.L., Eichholtz, D., Rogers, S.A., Fraley, R.T.: A simple and general method for transferring genes into plants. - Science 227: 1229-1231, 1985.

Lacerda, A.F., Vasconcelos, E.A., Pelegrini, P.B., Grossi de Sa, M.F.: Antifungal defensins and their role in plant defense. Front. Microbiol. 5: 116, 2014.

Lacerda, A.F., Del Sarto, R.P., Silva, M.S, De Vasconcelos, E.A.R., Coelho, R.R., Dos Santos, V.O., Godoy, C.V., Seixas, C.D.S, da Silva, M.C.M., Grossi-de-Sa, M.F.: The recombinant pea defensin Drr230a is active against impacting soybean and cotton pathogenic fungi from the genera Fusarium, Colletotrichum and Phakopsora. - 3 Biotech 6: 59, 2016.

Lay, F.T., Brugliera, F., Anderson, M.A.: Isolation and properties of floral defensins from ornamental tobacco and petunia. Plant Physiol. 131: 1283-1293, 2003.

Li, Z., Zhou, M., Zhang, Z., Ren, L., Du, L., Zhang, B., Xu, H., Xin, Z.: Expression of a radish defensin in transgenic wheat confers increased resistance to Fusarium graminearum and Rhizoctonia cerealis. - Funct. integr. Genomics 11: 63-70, 2011.

Li, H.L., Liu, D.Q., He, H., Zhang, N.N., Ge, F., Chen, C.Y.: Molecular cloning of a 14-3-3 protein gene from Lilium regale Wilson and overexpression of this gene in tobacco increased resistance to pathogenic fungi. - Sci. Hort. 168: 9-16, 2014.

Liu, D., He, X., Li, W., Chen, C., Ge, F.: A $\beta$-1,3-glucanase gene expressed in fruit of Pyrus pyrifolia enhances resistance to several pathogenic fungi in transgenic tobacco. - Eur. J. Plant Pathol. 135: 265-277, 2013a.

Liu, D., Li, W., He, X., Ding, Y., Chen, C., Ge, F.: Characterization and functional analysis of a novel PGIP gene from Pyrus pyrifolia Nakai cv Huobali. - Acta Physiol. Plant. 35: 12471256, 2013b.

Liu, D.Q., Han, Q., Shah, T., Chen, C.Y., Wang, Q., Tang, B.F., Ge, F.: A hybrid proline-rich cell-wall protein gene $J_{S P R P 1}$ from Juglans sigillata Dode confers both biotic and abiotic stresses in transgenic tobacco plants. - Trees 32: 1199-1209, 2018.

Livak, K.J., Schmittgen, T.D.: Analysis of relative gene expression data using real-time quantitative PCR and the 2- $\Delta \Delta C$ T method. - Methods. 25: 402-408, 2001.

Mello, E.O., Dos Santos, I.S., Carvalho, A.O., De Souza, L.S., De Souza-Filho, G.A., Do Nascimento, V.V., Machado, O.L., Zottich, U., Gomes, V.M.: Functional expression and activity of the recombinant antifungal defensin $P v D /$ r from Phaseolus vulgaris L. (common bean) seeds. - BMC Biochem. 15: 7, 2014.

Miao, Z.Q., Li, S.D., Liu, X.Z., Hen, Y.J., Li, Y.H., Wang, Y., Guo, R.J., Xia, Z.Y., Zhang, K.Q.: The causal microorganisms of Panax notoginseng root-rot disease. - Sci. agr. sin. 39: 1371-1378, 2006.

Nawrot, R., Barylski, J., Nowicki, G., Broniarczyk, J., Buchwald, W., Goździcka-Józefiak, A.: Plant antimicrobial peptides. Folia microbiol. (Praha) 59: 181-196, 2014.

Nguyen, N.N., Ranwez, V., Vile, D., Soulié, M.C., Dellagi, A., Expert, D., Gosti, F.: Evolutionary tinkering of the expression of $P D F 1$ s suggests their joint effect on zinc tolerance and the response to pathogen attack. - Front Plant Sci. 5: 70, 2014.

Odintsova, T.I., Slezina, M.P., Istomina, E.A., Korostyleva, T.V., Kasianov, A.S., Kovtun, A.S., Makeev, V.J., Shcherbakova, L.A., Kudryavtsev, A.M.: Defensin-like peptides in wheat analyzed by whole-transcriptome sequencing: a focus on structural diversity and role in induced resistance. - Peer J. 7: e6125, 2019.

Oomen, R.J., Séveno-Carpentier, E., Ricodeau, N., Bournaud, C., Conéjéro, G., Paris, N., Berthomieu, P., Marquès, L.: Plant defensin AhPDF1.1 is not secreted in leaves but it accumulates in intracellular compartments. - New Phytol. 192: 140-150, 2011.

Pervieux, I., Bourassa, M., Françoise, L., Hamelin, R., Armand, S.: A spruce defensin showing strong antifungal activity and increased transcript accumulation after wounding and jasmonate treatments. - Physiol. mol. Plant Pathol. 64: 331341, 2004.

Sarkar, P., Jana, K., Sikdar, S.R.: Overexpression of biologically safe Rorippa indica defensin enhances aphid tolerance in 
Brassica juncea. - Planta 246: 1029-1044, 2017.

Sasaki, K., Kuwabara, C., Umeki, N., Fujioka, M., Saburi, W., Matsui, H., Abe, F., Imai, R.: The cold-induced defensin TAD1 confers resistance against snow mold and Fusarium head blight in transgenic wheat. - J. Biotechnol. 228: 3-7, 2016.

Seo, H.H., Park, S., Park, S., Oh, B.J., Back, K., Han, O., Kim, J.I., Kim, Y.S.: Overexpression of a defensin enhances resistance to a fruit-specific anthracnose fungus in pepper. PLoS ONE 9: e97936, 2014.

Silverstein, K.A.T., Graham, M.A., Paape, T.D., Van den Bosch, K.A.: Genome organization of more than 300 defensin-like genes in Arabidopsis. - Plant Physiol. 138: 600-610, 2005.

Silverstein, K.A.T., Moskal, W.A., Wu, H.C., Underwood, B.A., Graham, M.A., Town, C.D., Van den Bosch, K.A.: Small cysteine-rich peptides resembling antimicrobial peptides have been under-predicted in plants. - Plant J. 51: 262-280, 2007.

Spelbrink, R.G., Dilmac, N., Allen, A., Smith, T.J., Shah, D.M., Hockerman, G.H.: Differential antifungal and calcium channel-blocking activity among structurally related plant defensins. - Plant Physiol. 135: 2055-2067, 2004.

Terras, F.R., Eggermont, K., Kovaleva, V., Raikhel, N.V., Osborn, R.W., Kester, A., Rees, S.B., Torrekens, S., Van Leuven, F., Van der Leyden, J.: Small cysteine-rich antifungal proteins from radish: their role in host defense. - Plant Cell 7: 573-588,
1995.

Tesfaye, M., Silverstein, K.A., Nallu, S., Wang, L., Botanga, C.J., Gomez, S.K., Costa, L.M., Harrison, M.J., Samac, D.A., Glazebrook, J., Katagiri, F., Gutierrez-Marcos, J.F., Van den Bosch, K.A.: Spatio-temporal expression patterns of Arabidopsis thaliana and Medicago truncatula defensin-like genes. - PLoS ONE 8: e58992, 2013.

Thomma, B.P., Cammue, B.P., Thevissen ,K.: Plant defensins. Planta 216: 193-202, 2002

Weerawanich, K., Webster, G., Ma, J.K., Phoolcharoen, W., Sirikantaramas, S.: Gene expression analysis, subcellular localization, and in planta antimicrobial activity of rice (Oryza sativa L.) defensin 7 and 8. - Plant Physiol. Biochem. 124: 160-166, 2018.

Zasloff, M.: Antimicrobial peptides of multicellular organisms. Nature 415: 389-395, 2002.

Zhang, J.S., Zhang, B.X., Du, M.M., Wang, X.Y., Li, W. Chinese preparation Xuesaitong promotes the mobilization of bone marrow mesenchymal stem cells in rats with cerebral infarction. - Neural Regen Res. 11: 292-297, 2016.

Zhang, N.N., Guan, R.P., Yang, Y., Bai, Z.W., Ge, F., Liu, D.Q.: Isolation and characterization of a Fusarium oxysporumresistant gene $L r G L P 1$ from Lilium regale Wilson. - In Vitro cell. dev. Biol. Plant. 53: 461-468, 2017. 